\title{
Entrenamiento muscular a través de tecnología isoinercial en un jugador defútbol profesional intervenido de rotura total de LCA. Estudio decaso. Muscle training using isoinertial technology in a professional footballer following surgery for a complete ACL tear. A case study. \\ Víctor Manuel Núñez Alvarez; Carolina Lancho Poblador; Juan Manuel Ramírez Pistón Universidad de Córdoba (España)
}

\begin{abstract}
Resumen. La incorporación del trabajo con tecnología isoinercial en los diferentes programas de entrenamiento ha contribuido a una notable mejora en el desarrollo de la fuerza y potencia muscular. Objetivos: evaluar la fuerza de la musculatura flexora y extensora de la rodilla en fase concéntrica y excéntrica. Método: durante 4 semanas (3 sesiones semanales) se ha realizado un programa de entrenamiento con tecnología isoinercial con movimientos simultáneos de flexión y extensión de rodilla de ambas extremidades. Antes y después del programa de entrenamiento se ha evaluado la potencia del tren inferior a través de la tecnología isoinercial versapulley en ejecuciones separadas de extensión y flexión de rodilla. Se ha obtenido y monitorizado datos de potencia, velocidad, fuerza, trabajo, tiempos de repeticiones,.., tanto en fase concéntrica como excéntrica. Resultados: se ha obtenido mejoras significativas $(p<0,05)$ tanto en fase concéntrica como excéntrica en movimientos de flexión y extensión de la rodilla. Conclusiones: durante las primeras etapas de la recuperación funcional los ejercicios generales provocan adaptaciones entre extremidades y disminuyen la asimetría provocada por la lesión deportiva. En un proceso de recuperación física disminuye la carga excesiva que provoca trabajar la extremidad por separado y en consecuencia disminuir el periodo de recuperación.
\end{abstract}

Palabras clave: lesiones, LCA, potencia, Entrenamiento isoinercial.

Abstrac: Training programmes incorporating isoinertial technology have contributed to a considerable improvement in developing muscle force and muscle power. Objectives: to evaluate knee flexor and extensor muscle force in concentric and eccentric contractions. Method: A 4-week training programme (3 sessions/week) using isoinertial technology was implemented, involving simultaneous flexion and extension of both knees. Before and after the training programme, lower-body power was evaluated using «VersaPulley» isoinertial technology in separate knee extension and flexion exercises. Data for power, velocity, force, work, repetition times etc. were recorded and monitored for both concentric and eccentric contractions. Results: Significant improvement $(\mathrm{p}=0.05)$ was recorded for both concentric and eccentric contractions in knee flexion and extension. Conclusions: In the early stages of functional recovery, general exercise enhances adaptation between limbs, reducing the asymmetry prompted by sports injuries. In the course of physical recovery, the overload caused by working separately with the affected limb is diminished, thus shortening recovery time. Key words. Injuries, ACL, power, isoinertial training.

\section{Introducción}

El fútbol es un deporte muy exigente funcional y fisiológicamente. A nivel competitivo, la sobrecarga de las estructuras anatómicas de las extremidades inferiores, puede incrementar el riesgo de sufrir una lesión muscular o articular (Inklaar, 1994). Las lesiones del ligamento cruzado anterior (LCA) son una de las más frecuentes y severas entre los jugadores de fútbol (Agel, Evans, Dick, Putukian, Marshall, 2007).

Esta patología, si no se diseña un programa de rehabilitación adecuado, se relaciona con importantes pérdidas funcionales, tales como un descenso en la velocidad de la contracción muscular, una disminución de la resistencia a la fatiga y un deterioro de la fuerza y el tono muscular (Álvarez et al., 2014; González et al., 2014).

El objetivo de las diferentes técnicas de reconstrucción y rehabilitación es la recuperación funcional de la articulación a niveles lo más cercano posibles al momento anterior a la lesión, en un plazo no superior a 6 meses desde la intervención (Saka, 2014). La reconstrucción quirúrgica del LCA produce buenos resultados en el $90 \%$ de los casos, siendo el rendimiento posterior similar al que había antes de la lesión (Erickson, 2014). Sin embargo, esto no garantiza la vuelta a la actividad deportiva de élite, por diferentes razones, como el fracaso del injerto, la inadecuada recuperación funcional y los factores psicológicos como el miedo a la recaída (Bauer, Feeley, Wawrzyniak, Pinkowsky, Gallo, 2014).

En el momento de regresar a la actividad deportiva, los atletas con una mayor debilidad muscular son los que presentan una mayor asimetría y alteración en la fuerza de contracción de los músculos (Schmitt, Paterno, Ford, Myer, Hewett, 2014; Schmitt, Paterno, Hewett, 2012; Vaquero, Martínez, Alacid, Ros, 2015). Estas circunstancias evidencian la necesidad de implementar programas de rehabilitación neuromuscular que tengan en cuenta estos desequilibrios y que se orienten a una recuperación funcional simétrica de ambas extremidades, incrementando la

Fecha recepción: 07-07-15- Fecha envío revisores: 07-07-15- Fecha de aceptación: 17-12-15 Victor Manuel Núñez Álvarez cm1nnalv@uco.es confianza de los atletas en la capacidad funcional de la rodilla.

El entrenamiento excéntrico ha demostrado ser de gran importancia para prevenir lesiones por su efecto sobre la musculatura y su capacidad para generar grandes tensiones (Askling, Karisson, Thorstensson, 2003; Brughelli et al., 2010; García, Ruiz, Latorre, Pedro, 2015), así como para el tratamiento de las lesiones de la rodilla (Bahr, Fossan, Loken, Engebreten, 2006).

Una modalidad de entrenamiento que puede reforzar el efecto de las acciones excéntricas y, en consecuencia, la fuerza, es el entrenamiento isoinercial (Norrbrand, Pozzo, Tesch, 2010; Pearson, Harridge, Grieve, Young, Woledge, 2001). Este tipo de ejercicio se está utilizando cada vez más en el ámbito de la rehabilitación deportiva, ya sea de forma exclusiva o en combinación con otros procedimientos de entrenamiento.

En este estudio de caso se pretende demostrar la eficacia de un programa de entrenamiento isoinercial sobre la potencia y la capacidad de trabajo de la musculatura flexora y extensora de la rodilla (cuádriceps e isquiotibiales) en fase concéntrica y excéntrica en movimientos combinados de flexión y extensión de la propia articulación, así como sobre la disminución de la asimetría producida por la lesión tras la reconstrucción del ligamento cruzado anterior en un futbolista de élite.

\section{Material y métodos}

\section{Descripción del caso}

Jugador de fútbol profesional de la Liga española, de 29 años de edad (estatura: $173.0 \mathrm{~cm}$; peso: $65.0 \mathrm{~kg}$; IMC: 21.7). El caso presentaba un diagnóstico clínico de inestabilidad anterior de la rodilla derecha, sensación de inseguridad al deambular, con por lo menos uno de los signos clínicos de lesión del LCA positivo (cajón anterior, Lachman o pivot-shift). Las pruebas diagnósticas de imagen (resonancia magnética) manifiestan una rotura parcial del LCA sin rotura de la cápsula articular.

Se decide intervenir quirúrgicamente para la reconstrucción artroscópica del LCA. El método utilizado es injerto de semitendinosorecto interno cuádruple fijado en fémur y tibia con tornillos de interferencia bioabsorbibles de ácido poliláctico y fosfato tricálcico. 
Tras la operación, el proceso de cicatrización durará de 10 a 12 semanas. Se coloca una media antitromboembólica hasta el muslo y se indica analgésico (cada 6horas). El paciente comienza la terapia física el mismo día de la cirugía, siendo el objetivo permitir el retorno a las actividades deportivas sin límite entre los 6 y 7 meses posteriores a la cirugía.

Se realizó un seguimiento del paciente durante 1 año. Se efectuaron controles al día siguiente de la cirugía, a los 8 y 15 días, fecha en que se retiraron los puntos de sutura, y luego se hicieron evaluaciones médicas cada 3 semanas los dos primeros meses post-operatorios y, cada 6 semanas,el resto del año.

Básicamente, la reeducación corresponde 4 fases: el postoperatorio inmediato, la fase de la reeducación propiamente dicha, la reeducación complementaria y el regreso a la actividad deportiva. Las intervención durante el proceso de rehabilitación se desarrolló de la forma descrita en la Tabla 1.



\section{Evaluación}

Concluido el período de readaptación funcional se inició el trabajo de adaptación física conducente a la reincorporación a los entrenamientos en grupo. En la semana 17 se consideró que el paciente cumplía satisfactoriamente los criterios de recuperación funcional establecidos para dicha fase, por lo que estaba preparado para comenzar con el programa de entrenamiento isoinercial. El paciente realizaba extensión y flexión completa de la articulación sin dolor, el paso estaba normalizado y había recuperado la sensación de estabilidad, pudiendo realizar ejercicios de impacto controlado. Las medidas pretest evaluaron el nivel de simetría en cuádriceps e isquiotibiales en potencia, velocidad y capacidad de trabajo en acción concéntrica y excéntrica. Para ello se aplicó la tecnología isoinercial Versapulley (polea cónica excéntrica) en ejecuciones separadas de extensión y flexión de la rodilla, evaluando ambas piernas individualmente. Para el registro y evaluación de datos se ha utilizado la tecnología/softwareSmartCoach ${ }^{\mathrm{TM}}$.

\section{Proceso}

Un total de 13 han sido las sesiones/entrenamientos realizadas (3 por semana: lunes, miércoles, sábado). En cada sesión el atleta ha realizado 2 ejercicios de tecnología isoinercial: yoyo squat y yoyo legcurl. La carga de trabajo ha sido de 6 series de 10 repeticiones máximas (a máxima velocidad) con carga inercial de 2 u.n, con pausas/intervalos de descanso de 3 minutos. Previamente el jugador realiza un calentamiento que consiste en 5 minutos de movilidad y activación articular/muscular en camilla, 5 minutos de carrera aeróbica y 2 series de 8 repeticiones en cada ejercicio a intensidad progresiva. Al igual que para la evaluación, durante el entrenamiento se ha utilizado la tecnología/ software_SmartCoach ${ }^{\mathrm{TM}}$. Simultáneamente, el paciente ha realizado ejercicios pliométricos, ejercicios para el fortalecimiento del tronco y cade- ras, y ha realizado carrera en distancias cortas y de forma progresiva.

Se han recogido datos relativos a potencia media (w), pico de potencia (w), tiempo por repetición (s), trabajo (J), velocidad media del volante de inercia (revol/s) y pico de velocidad del volante de inercia (revol/s) en acción concéntrica y excéntrica en los cuádriceps e isquiotibiales.

\section{Estudio estadístico}

Para el análisis de la efectividad pretest-posttest se ha aplicado la prueba t de Student para dos muestras independientes, ya que se han considerado ambas ejecuciones como no relacionadas, siendo la unidad experimental cada una de las ejecuciones. El tamaño del efecto se ha calculado como el coeficiente d de Cohen. Se ha estudiado la asimetría como el cociente de la diferencia entre la pierna sana menos la afectada con el valor de la pierna sana, expresado en porcentaje, de tal manera que valores positivos indican que la variable tienen un valor superior en la pierna sana. El cambio en asimetría se ha analizado en valores absolutos.Se ha tomado como valor de significación el $5 \%$.

\begin{tabular}{|c|c|c|c|c|c|c|}
\hline \multirow[b]{2}{*}{ nnc } & \multicolumn{2}{|c|}{ Right Leg } & \multirow[b]{2}{*}{$\begin{array}{c}\text { Change } \\
(\%)\end{array}$} & \multicolumn{2}{|c|}{ Left Leg } & \multirow[b]{2}{*}{$\begin{array}{c}\text { Change } \\
\text { (\%) }\end{array}$} \\
\hline & $\begin{array}{l}\text { Pretest } \\
\text { M Sd }\end{array}$ & $\begin{array}{c}\text { Posttest } \\
\text { M }+ \text { Sd }\end{array}$ & & $\begin{array}{l}\text { Pretest } \\
M+S d\end{array}$ & $\begin{array}{c}\text { Posttest } \\
M+S d\end{array}$ & \\
\hline Av Power (W) & $169.3 \pm 15.1$ & $193.5 \pm 15.5$ & $14.3^{\mathrm{c}}$ & $199.3 \pm 17.8$ & $209.6 \pm 26.9$ & 5.2 \\
\hline Peak Power (W) & $322.9 \pm 31.7$ & $340.3 \pm 31.6$ & 54 & $354.9 \pm 41.6$ & $372.8 \pm 51.6$ & 5.0 \\
\hline Time Rep (s) & $0.9 \pm 0.0$ & $0.7 \pm 0.0$ & $-15.5^{c}$ & $0.8 \pm 0.0$ & $0.7 \pm 0.0$ & $-5.9^{\mathrm{b}}$ \\
\hline Work (J) & $143.7 \pm 12.1$ & $139.0 \pm 11.3$ & -3.3 & $149.6 \pm 10.9$ & $147.2 \pm 10.6$ & -1.6 \\
\hline Av Vel (revol/s) & $4.4 \pm 0.2$ & $4.4 \pm 0.2$ & -0.3 & $4.4 \pm 0.1$ & $4.5 \pm 0.1$ & 0.8 \\
\hline Peak Vel (revol/s) & $6.3 \pm 0.3$ & $6.2 \pm 0.2$ & -1.4 & $6.5 \pm 0.2$ & $6.4 \pm 0.2$ & -0.6 \\
\hline
\end{tabular}

Tabla 3.

Medidas de potencia y trabajo en fase excéntrica en la pierna intervenida (right leg) y en la pierna sana (left leg) en los cuádriceps y cambio producido tras el entrenamiento isoinercial.

\begin{tabular}{|c|c|c|c|c|c|c|}
\hline \multirow{2}{*}{ Eccentric } & \multicolumn{2}{|c|}{ Right Leg } & \multicolumn{4}{|c|}{ Left Leg } \\
\hline & $\begin{array}{c}\text { Pretest } \\
\mathbf{M} \pm \text { Sd }\end{array}$ & $\begin{array}{c}\text { Posttest } \\
\text { M } \pm \text { Sd }\end{array}$ & $\begin{array}{c}\text { Change } \\
(\%)\end{array}$ & $\begin{array}{c}\text { Pretest } \\
\mathbf{M} \pm \mathbf{S d}\end{array}$ & $\begin{array}{l}\text { Posttest } \\
\text { M } \pm S d\end{array}$ & $\begin{array}{c}\text { Change } \\
(\%)\end{array}$ \\
\hline Av Power (W) & $168.6 \pm 27.0$ & $183.1 \pm 25.3$ & 8.6 & $188.4 \pm 23.1$ & $206.4 \pm 23.4$ & 9.6 \\
\hline Peak Power (W) & $487.7 \pm 65.0$ & $550.8 \pm 79.9$ & $12.9^{\mathrm{a}}$ & $492.1 \pm 50.5$ & $489.9 \pm 57.9$ & -0.5 \\
\hline Time Rep (s) & $0.9 \pm 0.1$ & $0.8 \pm 0.1$ & $-11.3^{b}$ & $0.8 \pm 0.0$ & $0.7 \pm 0.0$ & $-10.2^{c}$ \\
\hline Work $(J)$ & $143.9 \pm 12.0$ & $139.2 \pm 11.0$ & -3.3 & $149.7 \pm 10.7$ & $147.5 \pm 10.3$ & -1.5 \\
\hline Av Vel (revol/s) & $4.9 \pm 0.2$ & $4.9 \pm 0.2$ & 0.1 & $4.9 \pm 0.1$ & $4.8 \pm 0.2$ & -2.6 \\
\hline Peak Vel (revol/s) & $6.3 \pm 0.3$ & $6.2 \pm 0.2$ & -1.4 & $6.5 \pm 0.2$ & $6.4 \pm 0.2$ & -0.6 \\
\hline
\end{tabular}

\section{Resultados}

\section{Extensión rodilla/Cuádriceps}

La acción concéntrica ha aumentado de forma significativa los valores de potencia media y ha disminuido el tiempo por repetición en la pierna intervenida quirúrgicamente (tabla 2). La prueba d de Cohen indica un elevado tamaño del efecto en ambos casos $\left(\mathrm{d}_{\text {AvPower }}=1.6 ; \mathrm{d}_{\text {Time }}\right.$ =4.6). En la pierna no intervenida, sólo se ha producido un cambio significativo en el tiempo medio de repetición, que ha disminuido $\left(\mathrm{d}_{\text {Time }}\right.$ Rep $=1.4$ ). En ambas piernas parece observarse una tendencia a la disminución de la capacidad de trabajo, aunque no resulta significativa.

Durante la acción excéntrica (tabla3), en la pierna intervenida se ha observado un incremento del pico de potencia $\left(\mathrm{d}_{\text {Peak Power }}=0.9\right) \mathrm{y}$ un descenso en el tiempo de repetición ( $\mathrm{d}_{\text {Time Rep }}=1.3$ ). Sin embargo, en la otra pierna sólo se ha observado la disminución del tiempo de repetición $\left(\mathrm{d}_{\text {Time Rep }}=1.8\right)$. Es destacable también el porcentaje de aumento observado en la potencia media, cercano al $10 \%$ en ambos casos, pero que no ha resultado significativo desde el criterio estadístico.

Respecto a la asimetría en la fase concéntrica del ejercicio, en la figura 1 podemos observar que para la potencia media, que es mayor en la pierna sana, se ha visto reducida en un 7.4\%. La asimetría relativa al pico de velocidad ha disminuido un $0.3 \%$, siendo superior en la pierna sana antes y después de la aplicación del programa. También se ha producido una importante disminución en el tiempo de respuesta (11.6\%), que es mayor en la pierna intervenida. Sin embargo, en la capacidad de trabajo (1.6\%), velocidad media del volante (1.2\%) y pico de velocidad del volante ( $0.8 \%$ ) parece obtenerse una ligera tendencia 




Av Power Peak Power Time Rep (s) Work (J) Av Vel (s) Peak Vel (s) (W) (W)

Figura 1. Porcentaje de asimetría en pretest (gris claro-light gray) y posttest (gris oscuro-dark gray) en los parámetros de potencia y trabajo medidos en los cuádriceps en fase concéntrica.

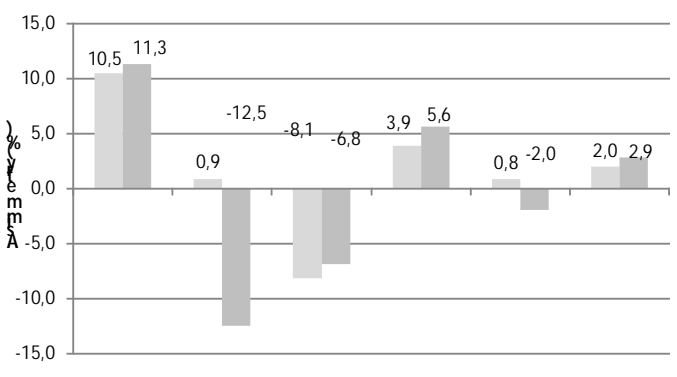

Av Power Peak Power Time Rep (s) Work () AvVel (s) Peak Vel (s) (W) $\quad(\mathrm{W})$

Figura 2. Porcentaje de asimetría en pretest (gris claro-light gray) y posttest (gris oscuro-dark gray) en los parámetros de potencia y trabajo medidos en los cuádriceps en fase excéntrica.

hacia el incremento de la asimetría, siendo todos estos valores superiores en la pierna sana tanto en el pretest como en el posttest.

En la fase excéntrica (Figura 2) se observa un notable incremento de la asimetría para el valor de pico de potencia(11.6\%), que era ligeramente superior en la pierna sana en el pretest, pero que, tras la intervención es notablemente mayor en la pierna operada. También ha crecido la asimetría en la potencia media (0.8\%), que es superior en la pierna sana. Para el tiempo de repetición, que es superior en la pierna afectada, se ha hallado una disminución de la asimetría (1.3\%), en el resto de parámetros de potencia parece observarse un ligera tendencia al incremento de la asimetría en acción excéntrica. La asimetría relativa a la capacidad de trabajo ha crecido un 1.7\%. Por último, también ha aumentado la asime-

Tabla 4

Medidas de potencia y trabajo en fase concéntrica en la pierna intervenida (right leg) y en la pierna sana (left leg) en los isquiotibiales y cambio producido tras el entrenamiento isoinercial.

\begin{tabular}{lcccccc}
\hline & $\begin{array}{c}\text { Right Leg } \\
\text { Pretest } \\
\text { Concentric }\end{array}$ & $\begin{array}{c}\text { Posttest } \pm \text { Sd } \\
\mathbf{M} \pm \text { Sd }\end{array}$ & $\begin{array}{c}\text { Change } \\
(\%)\end{array}$ & $\begin{array}{c}\text { Left Leg } \\
\text { Pretest } \\
\text { M } \pm \text { Sd }\end{array}$ & $\begin{array}{c}\text { Posttest } \\
\text { M } \pm \text { Sd }\end{array}$ & $\begin{array}{c}\text { Change } \\
(\%)\end{array}$ \\
\hline Av Power $(W)$ & $125.7 \pm 8.7$ & $130.4 \pm 16.3$ & 3.7 & $120.5 \pm 14.4$ & $126.1 \pm 17.4$ & 4.7 \\
Peak Power $(W)$ & $215.2 \pm 19.0$ & $228.9 \pm 33.8$ & 6.3 & $217.6 \pm 27.8$ & $213.7 \pm 25.5$ & -1.8 \\
Time Rep $($ s $)$ & $0.7 \pm 0.0$ & $0.9 \pm 0.0$ & $20.7^{\mathrm{c}}$ & $0.7 \pm 0.0$ & $0.9 \pm 0.0$ & $23.5^{\mathrm{c}}$ \\
Work $(J)$ & $90.8 \pm 4.1$ & $113.4 \pm 10.9$ & $24.9^{\mathrm{c}}$ & $89.3 \pm 8.9$ & $115.4 \pm 14.3$ & $29.3^{\mathrm{c}}$ \\
Av Vel (revol/s) & $3.5 \pm 0.1$ & $3.9 \pm 0.2$ & $13.0^{\mathrm{c}}$ & $3.4 \pm 0.3$ & $4.0 \pm 0.4$ & $17.0^{\mathrm{c}}$ \\
Peak Vel (revol/s) & $5.0 \pm 0.1$ & $5.6 \pm 0.3$ & $11.6^{\mathrm{c}}$ & $5.0 \pm 0.3$ & $5.7 \pm 0.4$ & $13.8^{\mathrm{c}}$ \\
\hline M: mean; Sd: Standard deviation; a $=\mathrm{p}<0.05 ; \mathrm{b}=\mathrm{p}<0.01 ; \mathrm{c}=\mathrm{p}<0.001$.
\end{tabular}

Tabla 5

Medidas de potencia y trabajo en fase excéntrica en la pierna intervenida (right leg) y en la piema sana (left leg) en los isquiotibiales y cambio producido tras el entrenamiento

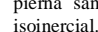

\begin{tabular}{lcccccc} 
isoinercial. & \multicolumn{1}{c}{ Right Leg } & \multicolumn{2}{c}{ Left Leg } \\
Eccentric & $\begin{array}{c}\text { Pretest } \\
\text { M } \pm \text { Sd }\end{array}$ & $\begin{array}{c}\text { Posttest } \\
\text { M } \pm \text { Sd }\end{array}$ & $\begin{array}{c}\text { Change } \\
\text { (\%) }\end{array}$ & $\begin{array}{c}\text { Pretest } \\
\text { M } \pm \text { Sd }\end{array}$ & $\begin{array}{c}\text { Posttest } \\
\text { M } \pm \text { Sd }\end{array}$ & $\begin{array}{c}\text { Change } \\
\text { (\%) }\end{array}$ \\
\hline Av Power $(W)$ & $116.0 \pm 11.6$ & $122.8 \pm 18.5$ & 5.9 & $109.8 \pm 16.8$ & $121.8 \pm 20.2$ & 10.9 \\
Peak Power $(W)$ & $284.6 \pm 45.9$ & $354.5 \pm 69.3$ & $24.6^{\mathrm{b}}$ & $272.6 \pm 60.0$ & $354.4 \pm 90.4$ & $30.0^{\mathrm{a}}$ \\
Time Rep $(\mathrm{s})$ & $0.8 \pm 0.1$ & $0.9 \pm 0.1$ & $18.3^{\mathrm{c}}$ & $0.8 \pm 0.1$ & $1.0 \pm 0.1$ & $16.5^{\mathrm{c}}$ \\
Work $(J)$ & $90.8 \pm 4.0$ & $113.5 \pm 10.9$ & $24.9^{\mathrm{c}}$ & $89.3 \pm 9.0$ & $115.4 \pm 14.4$ & $29.3^{\mathrm{c}}$ \\
Av Vel (revol/s) & $3.7 \pm 0.2$ & $4.3 \pm 0.3$ & $17.2^{\mathrm{c}}$ & $3.7 \pm 0.3$ & $4.4 \pm 0.4$ & $18.1^{\mathrm{c}}$ \\
Peak Vel (revol/s) & $5.0 \pm 0.1$ & $5.6 \pm 0.3$ & $11.6^{\mathrm{c}}$ & $5.0 \pm 0.3$ & $5.7 \pm 0.4$ & $13.7^{\mathrm{c}}$ \\
\hline M: mean; Sd: Standard deviation; a $=\mathrm{p}<0.05 ; \mathrm{b}=\mathrm{p}<0.01 ; \mathrm{c}=\mathrm{p}<0.001$.
\end{tabular}

tría de la velocidad media del volante de inercia (1.2\%), que en este caso ha cambiado de signo, ya que era superior en la pierna sana en el pretest, pero inferior en el posttest; así como en el pico de velocidad del volante (0.9\%), que es superior en la pierna sana antes y después de la intervención.

\section{Flexión rodilla/Isquiotibiales}

Durante la fase de flexión de rodilla y en la evaluación del efecto del programa sobre la potencia concéntrica medida en los isquiotibiales (tabla 4), el atleta ha aumentado el tiempo de repetición tanto en la pierna afectada $\left(\mathrm{d}_{\text {TimeRep }}=5.2\right)$ como en la sana $\left(\mathrm{d}_{\text {TimeRep }}=6.7\right)$. En ambas piernas, también se ha incrementado la capacidad de trabajo $\left(\mathrm{d}_{\text {Work }}=3.0\right.$ vs 2.3), la velocidad media del volante de inercia $\left(\mathrm{d}_{\mathrm{AvVl}}=2.8 \mathrm{vs} 1.7\right)$ y el pico de velocidad del volante de inercia ( $\mathrm{d}_{\text {Peak Vel }}=2.9 \mathrm{vs} 2.1$ ). Los valores de potencia media y pico de potencia parecen mostrar una tendencia a incrementarse tras la intervención en la pierna afectada, aunque no de forma estadísticamente significativa.

En la fase excéntrica (tabla 5), en la pierna afectada se ha producido un importante incremento del pico de potencia tras la intervención ( $\mathrm{d}_{\mathrm{Per}}$

$=1.2$ ). También ha aumentado de forma significativa la capacidad de trabajo $\left(\mathrm{d}_{\text {work }}=3.0\right)$, la velocidad media del volante de inercia $\left(\mathrm{d}_{\text {AvVel }}\right.$ $=2.6$ ) y el pico de velocidad del volante de inercia $\left(\mathrm{d}_{\text {Peak Vel }}=2.9\right)$. No obstante, tras la intervención también ha aumentado el tiempo de ejecución de cada repetición ( $\left.\mathrm{d}_{\text {Time Rep }}=2.2\right)$. La potencia media parece mostrar una tendencia a aumentar, aunque no de forma significativa. Los cambios producidos en la pierna no afectada han seguido la misma tendencia que en la pierna operada, con un incremento del pico de potencia $\left(\mathrm{d}_{\text {Peak Power }}=1.1\right)$, del tiempo de repetición $\left(\mathrm{d}_{\text {Time Rep }}=2.2\right)$, de la capacidad de trabajo $\left(\mathrm{d}_{\text {Work }}=2.2\right)$, así como de la velocidad media del volante de inercia $\left(\mathrm{d}_{\mathrm{Av} \text { Vel }}=2.2\right)$ y el pico de velocidad del volante de inercia $\left(\mathrm{d}_{\text {Peak Vel }}=2.1\right)$.

El análisis de la asimetría en la acción concéntrica muestra una reducción de la misma en los valores de potencia media (1.0\%), capacidad de trabajo (0.1\%), así como en velocidad media ( $0.2 \%)$ y pico de velocidad ( $0.3 \%$ ) del volante de inercia (Figura 3). La potencia media concéntrica era superior en la pierna afectada, sin que se haya observado un cambio de signo en el posttest. Sin embargo, la capacidad de trabajo, la media y pico de velocidad del volante ha cambiado de signo, reduciendo la asimetría, ya que en el pretest era superior en la pierna afectada, mientras que en el posttest estos valores son superiores en la pierna sana. Respecto al pico de potencia, se ha encontrado un incremento de la asimetría (6\%) acompañado de un cambio de signo, siendo superior en la pierna sana en el pretest e inferior en el posttest. En cuanto al tiempo de repetición, la asimetría ha aumentado un $2.2 \%$, siendo superior en la pierna sana en el pretest y posttest.

Durantela fase excéntrica (Figura 4), se observa una reducción de la asimetría en valores tales como potencia media $(4,8 \%$ ) y potencia pico $(4,4 \%)$, siendo superiores en la pierna operada en el pretest. De igual modo hay una disminución de asimetría en lo que al tiempo de repetición se refiere (3,5\%), siendo superior en el posttest a la pierna sana.

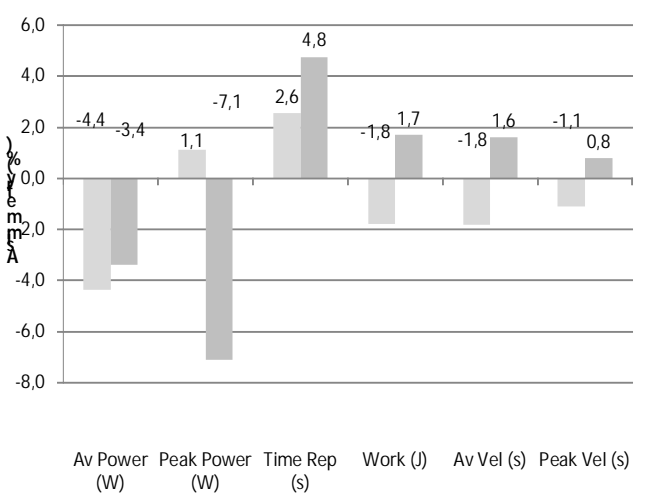

Figura 3. Porcentaje de asimetría en pretest (gris claro-light gray) y posttest (gris oscuro-dark gray) en los parámetros de potencia y trabajo medidos en los isquiotibiales en fase concéntrica. 




Av Power Peak Power Time Rep (s) Work (J) AvVel (s) Peak Vel (s) (W) (W)

Figura 4. Porcentaje de asimetría en pretest (gris claro-light gray) y posttest (gris oscuro-dark gray) en los parámetros de potencia y trabajo medidos en los isquiotibiales en fase excéntrica.

\section{Discusión}

El programa de entrenamiento isoinercial ha producido un efecto positivo sobre la potencia media en los cuádriceps (ejercicio Yo-Yo Squat) en acción concéntrica (extensión de la rodilla) en la pierna intervenida quirúrgicamente (change $=14.3 \%$ ), que se acompaña de un incremento de la velocidad de repetición. La potencia media de los cuádriceps de la pierna afectada en acción excéntrica, a pesar de haber aumentado en un $8.6 \%$, no ha resultado significativa estadísticamente, a pesar de que, según Norrbrand (2010), es en esta fase donde se produce la mayor adaptación electromiográfica atribuible al entrenamiento isoinercial. Sin embargo, consideramos este cambio relevante desde el punto de vista clínico, ya que supone una recuperación notable. El aumento de la potencia en los cuádriceps tras la intervención quirúrgica del LCA es uno de los indicadores más importantes para la recuperación funcional (Snyder-Mackler, Landin, Schepsis, Young, 1991), por lo que los resultados de este estudio reflejan la validez del ejercicio isoinercial para alcanzar este objetivo. La eficacia del entrenamiento inercial sobre la potencia ya había sido puesta de manifiesto en sujetos jóvenes sanos (Nack, Nack, Brzenczek-Owczarzak, Arlet, Adach, 2013).

El aumento de la potencia media no se observa con esta claridad en los isquiotibiales (ejercicio Yo-Yo Leg Curl), que en acción concéntrica solo aumenta un $3.7 \%$ y en excéntrica un $5.9 \%$, lo que viene a indicar que el efecto del entrenamiento isoinercial sobre la potencia sostenida a lo largo de las diferentes repeticiones solamente se produce a nivel del cuádriceps, especialmente en la fase concéntrica de la repetición, en el caso de la pierna operada. La débil adaptación de la potencia en los isquiotibiales podría deberse a la extracción de tejido tendinoso de este músculo para injertarlo en la rodilla, por lo que el tendón tardaría unos 18 meses en recuperar sus facultades normales (Carofino, Fulkerson, 2005). En esta pierna también se ha producido un incremento del pico de potencia de los cuádriceps y los isquiotibiales durante la correspondiente fase excéntrica (12.9\% y 24.6\%, respectivamente), demostrándose la capacidad del entrenamiento isoinercial de llevar a la pierna afectada a niveles puntuales más altos de potencia en dicha fase. El incremento de la potencia media en los cuádriceps en la fase concéntrica podría estar relacionado con un descenso en el tiempo de repetición (15.5\%), efecto que también se observa en la fase excéntrica (-11.3\%). Sin embargo, el efecto del entrenamiento isoinercial sobre este parámetro en los músculos isquiotibiales se produce en sentido contrario, con un aumento significativo del tiempo de repetición tanto en fase concéntrica (20.7\%) como excéntrica (18.3\%). Relacionado con esto se produce el hallazgo, aparentemente contradictorio, por el que a pesar de haber aumentado el tiempo de repetición, también se ha observado un incremento en la velocidad del volante de inercia, tanto en acción concéntrica como en excéntrica. Esta circunstancia se podría explicar por la relación dinámica entre el giro del volante y la aplicación de la fuerza necesaria para invertir la dirección del giro. En los isquiotibiales la fuerza de aceleración y desaceleración se aplica de forma progresiva durante la repetición, en una adaptación que desarrolla la capacidad de trabajo, lo que indica un movimiento menos explosivo que en los cuádriceps; por el contrario, en los cuádriceps se produce fundamentalmente una adaptación en la potencia, realizando el mismo trabajo en menos tiempo como consecuencia de una aceleración más explosiva, por lo que el volante de inercia mantiene su velocidad. Estos resultados podrían indicar que esta modalidad de entrenamiento está especialmente orientada a la mejora de lapotencia del músculo extensor dela articulación dela rodilla (cuádriceps), así como que la modalidad Yo-Yo Squat es capaz de movilizar fibras musculares rápidas en los cuádriceps, frente a la modalidad Yo-Yo Curl, que potencia la capacidad de trabajo en detrimento de la velocidad en los isquiotibiales. Si se examina el efecto sobre la capacidad de trabajo, se observa que el cambio es prácticamente nulo en los cuádriceps, pero muy importante en los isquiotibiales, que tanto en la flexión como en la extensión incrementan la energía necesaria para desplazar el volante de inercia en un 24.9\%. Por lo tanto, ambos ejercicios (Yo-Yo Squat y YoYo Curl) se presentan como complementarios en la rehabilitación de la musculatura flexora-extensora de la rodilla, ya que parecen trabajar aspectos específicos del binomio potencia-trabajo.

El trabajo para equilibrar los grupos de músculos agonistas y antagonistas en la pierna operada, reduciendo la asimetría con la pierna sana, es esencial para la recuperación del deportista, especialmente en el autoinjerto con tendones de isquiotibiales (Ageberg, Ross, Silbernagel, Thomeé, Roos, 2009). En la pierna no operada, las adaptaciones de los cuádriceps han sido de menor magnitud que en la pierna intervenida quirúrgicamente, aunque el sentido de las adaptaciones es similar, produciéndose un incremento de la potencia media reduciéndose el tiempo de repetición. En los isquiotibiales, las adaptaciones de la pierna sana se han producido en el mismo sentido y magnitud que en la pierna operada.

El entrenamiento isoinercial en cuádriceps ha producido una disminución de la asimetría en los valores de potencia media y en tiempo de repetición en la fase concéntrica. El aumento de la potencia de los cuádriceps, según estudios recientes, está relacionado con una mayor simetría en el movimiento y a un mejor rendimiento funcional (PalmieriSmith, Lepley, 2015).Sin embargo, se ha producido un incremento de la asimetría en el pico de potencia en la fase excéntrica, lo que puede estar ocasionado por un mayor control del movimiento en la pierna operada durante la flexión de rodilla con el fin de evitar nuevos daños, siguiendo la hipótesis de la Arthrogenic Muscle Inhibition (AMI) (Hart, Pietrosimone, Hertel, Ingersoll, 2010). En los isquiotibiales se ha hallado un aumento de la asimetría en el pico de potencia en la fase concéntrica (extensión de la rodilla), que en el posttest es netamente superior en la pierna afectada, lo que se interpretaría también como una adaptación por la cual el movimiento se hace más controlado por miedo a sufrir una lesión en un acto de naturaleza más explosiva, lo que lleva a producir un pico de potencia más alto. Sin embargo, en la fase excéntrica sí se ha producido una mejora manifiesta en la asimetría evaluada por la potencia media y el pico de potencia, que en el posttest se han igualado en las dos piernas.

El sentido de estas adaptaciones muestra la importancia que en el proceso de rehabilitación tiene el trabajo de la propiocepción (equilibrio o estabilidad), ya que la percepción de una rodilla inestable puede dar lugar a adaptaciones diferenciadas en la pierna sana y en la intervenida que pueden finalizar en una nueva lesión. El inicio del trabajo de fuerza debe iniciarse en la fase apropiada. Un inicio demasiado temprano puede ocasionar adaptaciones peculiares en ambas piernas, mientras que un comienzo tardío retrasaría todo el proceso de vuelta a la competición. La vuelta al trabajo sobre el campo es muy importante para devolver al deportista la confianza en la rodilla operada para rendir al mismo nivel que la rodilla sana, reduciendo de esta forma la asimetría que puede haberse generado por una adaptación diferente durante el período de rehabilitación.

\section{Conclusión}

El entrenamiento isoinercial en el proceso de recuperación de la potencia de los cuádriceps e isquiotibiales se ha mostrado como un método eficaz para conseguir su objetivo, tanto en la fase concéntrica 
como excéntrica de la flexión-extensión de la rodilla. Los dos grupos antagonistas de músculos examinados manifiestan adaptaciones complementarias, con una orientación a la potencia en los cuádriceps y a la capacidad de trabajo en los isquiotibiales. La reducción de la asimetría se ha comprobado como un proceso más complejo en el que puede jugar un papel importante la confianza del jugador a la hora de aplicar la fuerza, y que va a provocar que en la pierna afectada se realice una ejecución más controlada del movimiento de flexión o extensión.

\section{Referencias}

Ageberg, E. Ross, H.P. Silbernagel, K.G. Thomeé, R. Roos, E.M.(2009). Knee extension and flexion muscle power after anterior cruciate ligament reconstruction with patellar tendon graft or hamstring tendons graft: a cross-sectional comparison 3 years post surgery. Knee Surg Sports Traumatol Arthrosc, 17(2), 162-169.

Agel, J. Evans, T.A. Dick, R. Putukian, M. Marshall, S.W. (2007). Descriptiveepidemiology of collegiatemen's soccer injuries: National CollegiateAthletic Association Injury Surveillance System, 19881989 through 2002-2003, J Athl Train, 42, 270-277.

Álvarez Díaz, P. Alentorn Geli, E. Ramon, S. Steinbacher, G. Rius, M. Seijas, R. Ballester, J. Cugat, R. (2014). Effects of anterior cruciate ligament injury on neuromuscular tensiomyographic characteristics of the lower extremity in competitive male soccer players. Knee Surg Sports Traumatol Arthrosc, 23(11), 3407-13.

Askling, C. Karisson, J. Thorstensson, A. (2003). Hamstring injury occurrence in elite soccer players after preseason strength training wiht eccentric overload. Scand J Med Sci Sports, 13, 244-250.

Bahr, R. Fossan, B. Loken, S. Engebreten, L. (2006). Surgical Treatment Compared with Eccentric Training for Patellar Tendinopathy (Jumper’s Knee). J Bone Joint Surg Am, 88, 1689-1698.

Bauer, M. Feeley, B.T. Wawrzyniak, J.R. Pinkowsky, G. Gallo, R.A. (2014). Factors Affecting Return to Play After Anterior Cruciate Ligament Reconstruction:AReview of the Current Literature. Phys Sportsmed, 42(4), 71-79.

Brughelli, ., Mendiguchia, J. Nosaka, K. Idoate, F. Los Arcos, A. Cronin, J. (2010). Effects of eccentric exercise on optimum length of the knee flexors and extensors during the preseason in professional soccer players. Phys Ther Sport, 11, 50-55.

Carofino, B. Fulkerson, J. (2005). Medial hamstring tendon regeneration following harvest for anterior cruciate ligament reconstruction: fact, myth, and clinical implication. Arthroscopy, 21, 1257-1265.

Erickson, B.J. Harris, J.D. Heninger, J.R. Frank, R. Bush Joseph, C.A. Verma, N.N. Cole, B.J. Bach, B.R. (2014). Performance and Returnto-Sport After ACL Reconstruction in NFL Quarterbacks. Orthopedics, 37(8), e729-e734.

García, F. Ruiz, A. Latorre, R. Pedro, A. (2015). Influencia del puesto específico en la potencia y agilidad de jóvenes. Retos. Nuevas tendencias en educación física, deporte y recreación. 27, 58-61.

González, C. Del Coso, J. Abián-Vicén, J. López del Campo, R. Gutiérrez, D. Salinero, J. J. (2014). Cambios en la fuerza de miembros inferiores tras un mesociclo de pretemporada en futbolistas semiprofesionales. Retos. Nuevas tendencias en educación física, deporte y recreación. 26, 52-55.

Hart, J.M. Pietrosimone, B. Hertel, J. Ingersoll C.D. (2010). Quadriceps Activation Following Knee Injuries: A Systematic Review. J Athl Train, 45(1), 87-97.

Inklaar, H. (1994). Soccer injuries I. Incidence and severity. Sports Med, 18, 55-73.

Nack, M. Nack, A. Brzenczek-Owczarzak, W. Arlet, J. Adach, Z. (2013). Impact of inertial training on strength and power performance in young active men. J Strength Cond Res, 20, [Epub ahead of print].

Norrbrand, L. Pozzo, M. Tesch, P.A. (2010). Flywheel resistance training calls for greater eccentric muscle activation than weight training. Eur J Appl Physiol, 110, 997-1005.

Palmieri-Smith, R.M. Lepley, L.K. (2015). Quadriceps Strength Asymmetry After Anterior Cruciate Ligament ReconstructionAlters Knee Joint Biomechanics and Functional Performance at Time of Return to Activity. Am J Sports Med. 16. [Epub ahead of print].

Pearson, S.J. Harridge, S.D. Grieve, D.W. Young, A. Woledge, R.C. (2001). A variable inertial system for measuring the contractile properties of human muscle. Med Sci Sports Excer, 33(12), 20722076.

Saka, T. (2014). Principles of postoperative anterior cruciate ligament rehabilitation. World J Orthop, 5(4), 450-459.

Schmitt, L.C. Paterno, M.V. Ford, K.R. Myer, G.D. Hewett, T.E. (2014). Strength Asimmetry and Landing Mechanisms at Return to Sport after ACL Reconstruction. Med Sci Sports Sc,. [Published Ahead of Print].

Schmitt, L.C. Paterno, M.V. Hewett, T.E. (2012). The impact of quadriceps femoris strength asymmetry on functional performance at return to sport following anterior cruciate ligament reconstruction. J Orthop Sports Phys Ther, 42, 750-759.

Snyder-Mackler, L. Landin, Z. Schepsis, A.A. Young, J.C. (1991). Electrical stimulation of the thigh muscles after reconstruction of the anterior cruciate ligament. Effects of electrically elicited contraction of the quadriceps femoris and hamstring muscles on gait and on strength of the thigh muscles. J Bone Joint Surg, 73A, 1025-1036.

Vaquero, R. Martínez, I.Alacid, F; Ros, E. (2015). Efectos dela lateralidad sobre la flexibilidad, la fuerza-resistencia y el equilibrio en mujeres mayores activas. Retos. Nuevas tendencias en educación física, deporte y recreación. 27, 127-130.

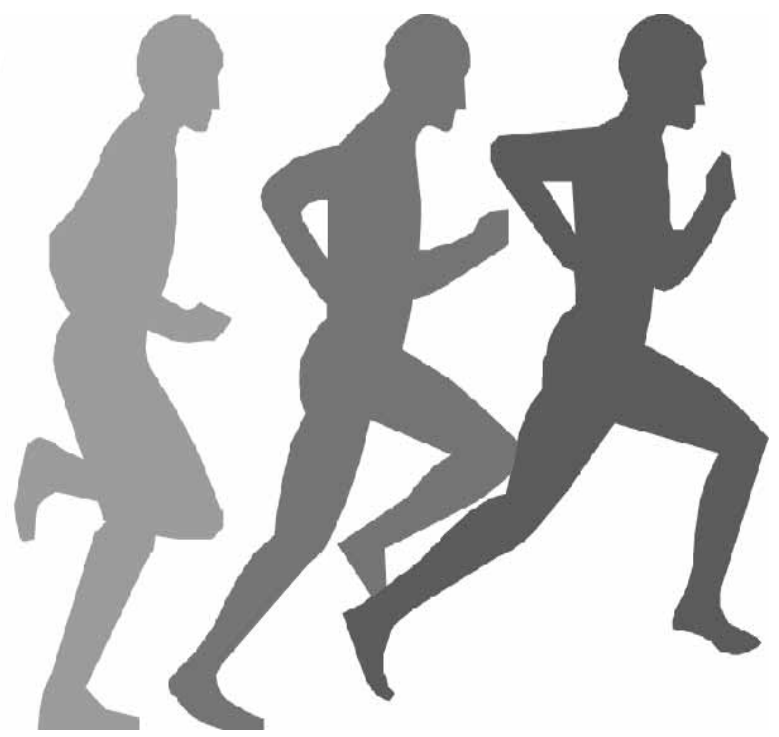

